\title{
Construction and Testing of the SLD Cerenkov Ring Imaging Detector*
}

M. Cavalli-Sforza. P. Coyle. D. Coyne, P. Gagnon. D A Williams! P Zucchelin ${ }^{2}$ Santa Cruz Institute for Partjcle Physics, tniversity of Californa Santa Cruz. Ca 95064

J. S. Whitaker, R. J. Wilson

Department of Physics. Boston tinitrrsity. Boston. MA 02215

\author{
A. Bean. D. Caldwell. J. Dubosty. J. Huber, A. Lu, I Mathys. S McHugh. A Morrison. \\ M. Witherell. S. Yellin \\ Department of Physics, l'niversity of California, Santa Barbara. CA 93106
}

R. A. Johnson, J Martinez, B. Meadows. M. Mussbaum. A. X. S. Santha. A Shoup. I Stockdale

Department of Physics, l'niversity of Cincinnati. Cincinnatı. OH $\$ 5221$

P. Jacques, R. Plano, P. Stamer

Serin Physics Laboratory, Rutgers liniversity, P.O Box 849. Piscataway, NI 08855

\author{
P: Antilogus, D. Aston, T. Bienz, F. Bird S. Dasu, W Dunwoodie. G Hallewell. \\ H. Kawahara. Y. Kwon, D. Leith. D. Muller, T. Nagamine. I. Pavel. B Rateljf. P Rensing. \\ D. Schultz, S. Shapiro. C. Simopoulos. E. Solodovi X. Toge. J. Va vra. S Williams \\ Stanford Linear Accelerator Center. Stanford t'niversity; Stanford. CA 94309 \\ K. Abe, K. Basegawa. F. Suekane. H. Yuta \\ Department of Physics, Toholu Vniversity; Aramaki. Senda 980. Japan
}

\begin{abstract}
We report on the construction of the Cerenkor Ring Imagjng Detector (CRJD) for the SLD experiment at the SLAC Linear Collider and the testing of its components. We include results from testing the drift boxes, liquid radiator trays, and mirrors for the barrel CRID. We also discuss development of the support systems essential for the operation of the CRID: gas and liquid recirculator systems and monitoring.
\end{abstract}

\section{Introduction}

A large Cerenkov Ring Imaging Detector (CRID) $[1,2]$ is cursently under construction for the SLD experiment at the SLAC Linear Collider (SLC). The barrel CRID will peovide almost complete particle identification over the central $70 \%$ of the solid angle (figure 1). By making use of

\footnotetext{
-Writk mupported by the Department of Enercy, contracts DEAC03-765FO051s and DE-AT03-79ER70023 and by the National Science Foundation under Grants PHY 88-13669 and PHY88-13018. ispenker

i Viejting from Intituto di Fisica, Univenaite de Ferrara, 1.44100 Fertara, Iealy

Srement Addrew: EP Divition, CERN, CH-1211. Geneva 23, Switzerland.

Termanent Addrew: Inctitute of Nuclear Physics, Novotibinal. 630090. USSR.
}

both liquid and gaseous radiators. $\pi / \mathrm{h} / \mathrm{p}$ separation will be possible up to about $30 \mathrm{GeV} / \mathrm{c}$. and $\mathrm{e} / \pi$ separation up to about $6 \mathrm{GeV} / \mathrm{c}$. Endcap CRID detectors are currently under design; in this paper we restrict our discussion to the barrel.

The barrel is constructed with a geometry of ten azimuthal sectors on each side of the mid-plane. In each sector, there are iwo driff boxes. each coupled to a multiwire proportional chamber sensitive to single electrons. or "single-electron detector" [3]. The drift box and detector combination serves to image Ćerenkov lighı produced in the radiators. A Cerenkov photon with wavelength less than $230 \mathrm{~nm}$ entering a drift box can ionize the drift gas mixture containing about 0.1\% TMAE (tetrakis[dimathylamino]ethylene). giving a single photoelectron. (To be transparent to photons of this short wavelength, the drift box windows are made of fused silica. t.e. quartz.) The resulting photoelectron drifts up to 1.27 meters in a uniform eleetric field which is nearly parallel to the magnetic field in a time projection geonetry. It is detected by a plane of ninety-three $7 \mu \mathrm{m}$ diameter carbon sense fibers, spaced at a pitch of $3.2 \mathrm{~mm}$, in the detector. The electronics used to read out the detector are discussed elsewhere [j]. The three production coordinates of the eles. tson are measured from the drift time, the position of the fiber giving the signal, and charge division along the fiber. The production coordinates are used together with track- 


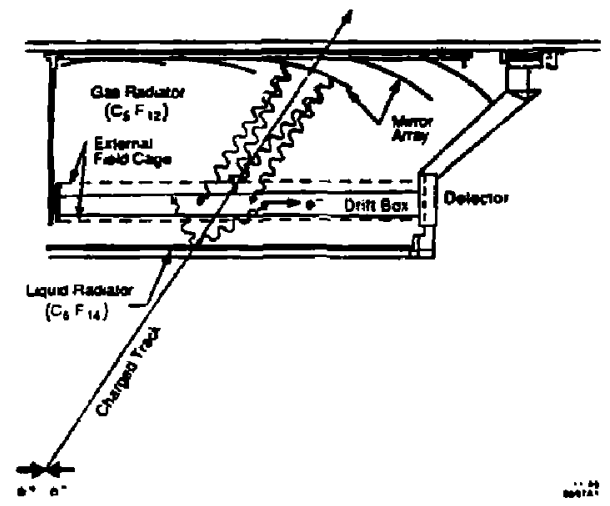

Figure 1: Principal Components of the SLD CRID Barrel system.

ing information from the SLD central drift chamber [1] to determine the Cerenkov angle and thus particle mass.

The liquid radiator is a $1 \mathrm{~cm}$ thick layer of $\mathrm{C}_{6} F_{14}$, contained in a G-10 tray with a quartz window. The quartz window is on the side away from the beam axis, allowing the Cerenkov radiation produced in the liquid to reach a drift box for detection. There are forty $t ; s$ in the finished barrel, one corresponding to each drift box.

The gas radiator is obtained by filling the CRID vessel with gaseous $\mathrm{C}_{3} \mathrm{~F}_{12}$. Cerenkov photons produced in the gas are focused on the drift boxes by spherical mirrors mounted on the onter wall of the CRUD vessel. Each drift box detects light from two rows of five misrors. The $\mathbf{4 0 0}$ mitrors in the barrel give complete coverage.

The CRID is operated at $40^{\circ} \mathrm{C}$ in order to keep the $\mathrm{C}_{5} \mathrm{~F}_{12}$, which boils at $30^{\circ} \mathrm{C}$, in the gaseous phase and to prevent condensation of TMAE in the drift box gas. The drift gas is saturated with TMAE at $28^{\circ} \mathrm{C}$ by bubbling the gas through liquid TMAE maintained at that temperature.

\section{Production Status}

The mechanieal structure, or vessel, of the barrel CRID has been installed in SLD. All $\mathbf{4 0 0}$ misrors have been mounted, atigned, and installed in the vessel. A final survey of the mirrors positions and orientations is in progress. Before it was moved into SLD, the vessel was also fitted with the radiatot gas temperature control system and central HV distribution plane.

About $80 \%$ of the drift boxes for the barrel CRID are finished, and assembly of the single-electron detectors is complete. Almost two thirds of the liquid radiator trays are finished. Finished drift boxes, detectors, and radiator trays are stoted in a clean room with the temperature con-

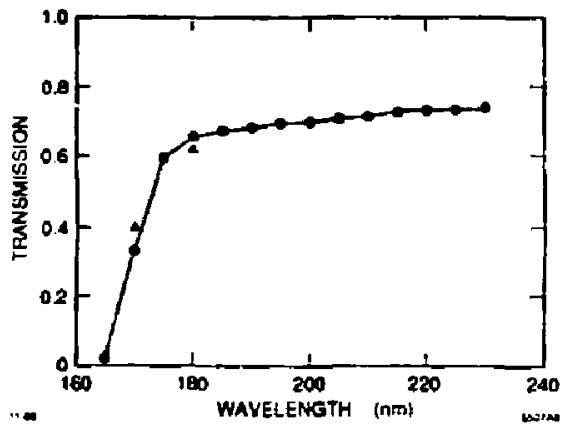

Figure 2: Transmission of a completed drift box. The circles are the measured values. The triangles are the estimate from the transmission of the individual windows. including losses from the field-shaping wises.

trolied to $37^{\circ} \mathrm{C}$ and a relative humidity of less than $10 \%$. The room is equipped so that the interior of the drift boxes and radiator trays can be purged with dry. boil-off nitrogen.

\section{Component Testing}

\section{Drift bores}

In order for the drift boxes to detect Cerenkov phorons from the radiators with high efficiency. theee items are critical to their performance. The quartz windows must have good transmission for wavelengths short enough to ionize the TMAE in the drift box. Second, the resulting photoelectrons must have a long lifetime i 100-200 $\mu \mathrm{s}$ ) against reattachment, giving them time to drift to the deteetor. Finally, the drift field must be unifotm so that the production poiat of an electron can be reconstructed with millimeter accuraty from the signal observed by the single-election detector.

The UV transparency of all quartz sheets arriving from the manufacturer is measured in a vacuum test station This device allows a UV beam to be scanned over the whole $125 \mathrm{~cm} \times 30 \mathrm{~cm}$ surface of an assembled window or a completed drift box. Figure 2 shows the transmission measured at one point for a completed drift box. It is in good agreement with the transmission predicted from the measurements of the individual windows and losses due to the field-shaping wires on the quartz surface, indicating that the assembly process does not degrade the transmission of the windows. Measurements taken at many posj$t$ :uns on the box indicate good uniformity of transmission over the surface.

Finished drift hoxes are fitted with a single-electron de- 


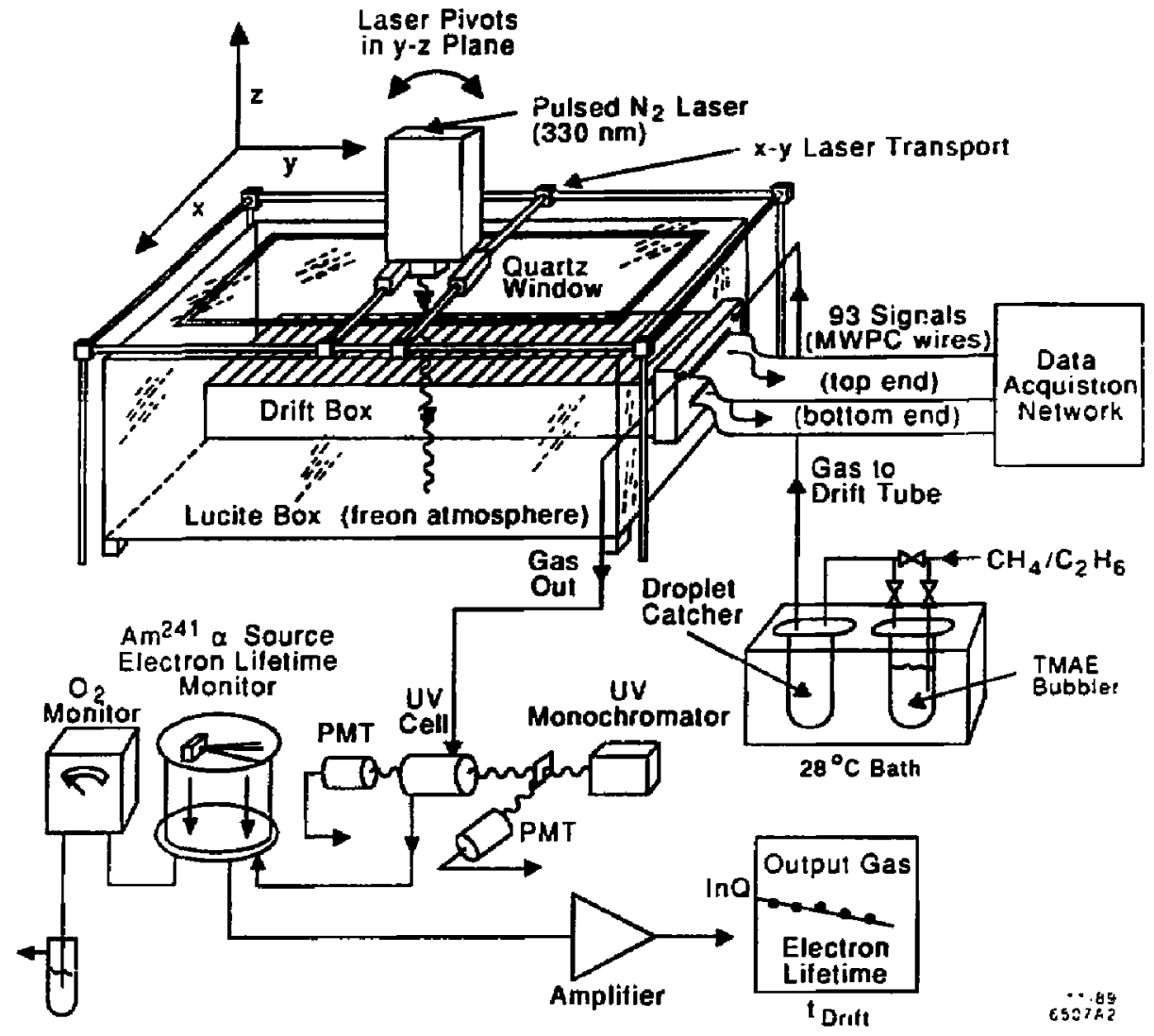

Figure 3: The test apparatus for the measurement of photoelectron drift distortion, electron lifetime, and drift gas $\mathbf{L V}$ transparency. The distortion measurements of Figures 4-6 were made with this apparatus.

tector and tested in a high voltage set-up which elosely simulates the electrostatic configuration of the actual CRID vessel. Here we test the high voltage performance only. The detector has first been tested in a fixture described in $[3]$.

The drift box and detector assembly then undergoes further testing in the apparatus of figure 3 . The assembly is placed in a Lucite test enclosure and connected to a gas system which provides drift gas either with or without TMAE. The Lucite enclosure can be filled with Freor to help the drift box hold high voltage and to test for Freon leakage into the drift volume. For these tests we use the Freon, $\mathrm{C}_{2} \mathrm{Cl}_{2} \mathrm{~F}_{4}$. The output gas can be monitored for electron lifetime [7] and UV transparency. UV absorption in the $160-23 \mathrm{~J} \mathrm{~nm}$ region is a sensitive check of the TMAE concentration.

To maintain a long lifetime against electron reattachment, the drift gas must be of high purity. Not only must pure gas be delivered to a drift box by the gas system, but it must remain pure inside the box. (The gas flow is about one volume change every two hours.) To prevent the electronegative $\mathrm{C}_{5} \mathrm{~F}_{12}$ of the gas radiator from getting into a drift box, each finished drift box is leak checked with a helium leak detector to a sensitivity of $10^{-5} \mathrm{~cm}^{3} / \mathrm{s}$. The materials used in the construction of the drift box have been carefully selected to minimize reaction with the TMAE in the drift gas (see [6]).

The electron lifetime monitor on the output gas has been useful for comparing relative lifetime but has been difficult to calibrate absolutely. We have recently measured the electron lifetime directly in a drift box. The apparatus in figure 3 includes a pulsed $\mathbf{N}_{2}$ laser (wavelength $=\mathbf{3 3 7}$ nm) mounted on an $x-y$ transport allowing it to be pointed into any region of the drift bux. The laser shines into the drift box through a quartz window and jonizes the drift gas via a double-photon process. By firing the laser into the box at several difterent drift distances and examining the number of observed electrons versus drift distance. we 


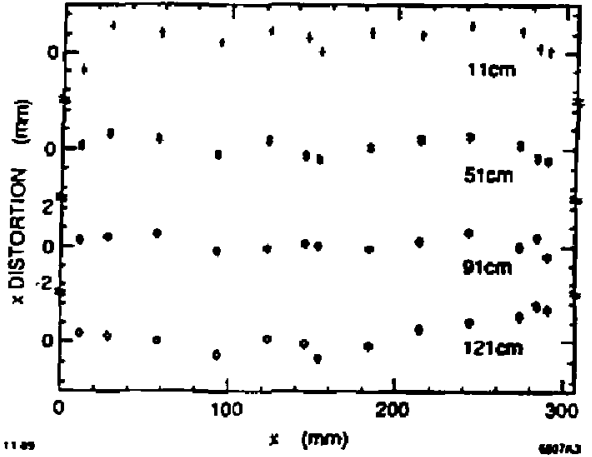

Figure 1: Difference between the expected and measured $\boldsymbol{x}$ position for different values a $x$ across the width of CRID drift box number 6. Data are shown for four different drift distances, ranging from 11 to $121 \mathrm{~cm}$.

have measured a lifetime of approximately $100 \mu \mathrm{s}[8]$. This is satisfactory for drift box operation.

The laser can also be used to measure distortions in the trajectories of drifting electrons by comparing the reconstructed light spots with their known positions (see [B]). (Althougb each drift box is equipped with gating electrodes to suppress the entry of positive ions into the drift volume [4], measurements done so far have been without gating.) Distortions in the $x-y$ plane, the plane parallel to the windows, are measured by firing the laser vertically into the drift box. The laser intensity is adjusted so that the mean number of photoelectrons produced by one pulse is $\sim 0.1$. At this intensity, events with multiple photoelectrons are rare. Upon reaching the detector, the electron produces a signal on a single wire. Taking many laser pulses in the same position, the hits are distributed over two to four wires because of diffusion and the finite spot size from the leser. This allows the mean position to be determined to better than the wire pitch. Comparing the reconstructed wire address witi the address that is predicted from the laser position, assuming a uniform drift field, measures the extent to which the drift field is not uniform.

Distortion measurements have been made in this way on several drift boxes. Figure 4 shows distortions measured across the wjdth of a drift box, for several drift distances. The plol shows the measured position minus the predicted position, in mm. The distortions are largest near the G-10 side walla, for $x$ near 0 and $300 \mathrm{~mm}$, but even there they do not exceed $2 \mathrm{~mm}$.

Distortions in the direction normal to the window, paraljel to the wires in the detector, can also be investigated. This is done by pivoting the laser so that it shines into the hox at an angle, typically $45^{\circ}$. The electrons are observed

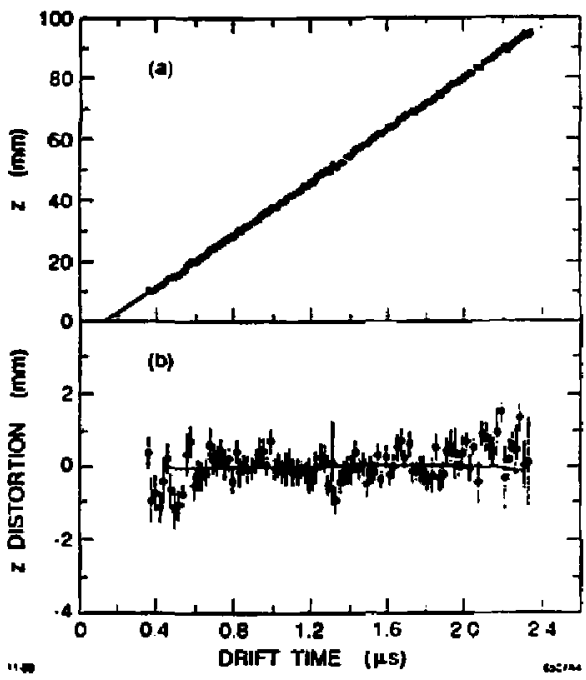

Figure 5: Use of an angled laser track to investigate : distortions. (a) Reconstructed $z$ position as a function of drift time (the zero for the time coordinate is arbit rary). A line with slope predieted from the laser angle and the drift velocity is superimposed on the data. (b) The residuals to the line shown in part a. The curve shows the residuals predicted by an electrostatic simulation. These results are for the normal operating potentials.

on a few neighboring wires, as before, but now the drift time varies depending on the depth $(z)$ of ionization. The apparent $z$, as observed at the detector, is reconstructed by charge division. We djvide the measured drift time of the observed signals into 20 ns bins, and in each bin we calculate the mean measured 2 . Figure 5a shows data on the resulting mean $z$ versus drift time. We fit the data to a straight line with the slope fixed by the known laser angle and measured drift velocity. The residuals (or distortions) are shown in figure $\mathbf{5 b}$. The measurements we show here are taken at the center of the detector, t.e., equidistant from the two side walls. Similar measurements 1.5 $\mathrm{cm}$ from the side wall show distortions of up $105 \mathrm{~mm}$ in the corners of the drift volume, with $2 \mathrm{~mm}$ being a more typical value.

One important source of possible distortions in the drift field is a mismatch of potentials in the transition region between the drift volume and the detector. We have investigated this by varying the potential of the drift box (keeping the gradient of the drift field within the box constant at $400 \mathrm{~V} / \mathrm{cm}$ ) while keeping the detector at the standard operating voltages. The results shown in figures 4 and 5 were obtained at the optimum voltages as determined both from 


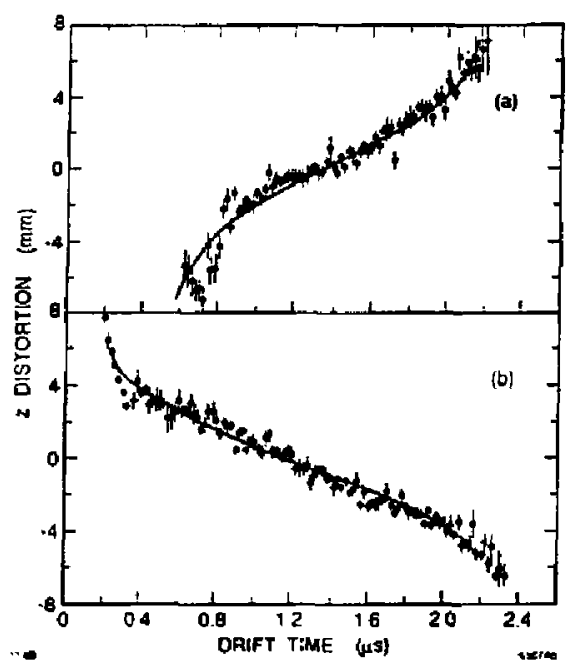

Figure 6: Effect of potential matching on z reconstruction. These plots are analogous to figure $5 \mathrm{~b}$. They result from decreasing ( $a$ ) and increasing (b) the potential difference between the drift box and the detector by $200 \mathrm{~V}$. The curves are the predictions of the electrostatic simulation.

the data and from electrostatic simulations [9]. The residuals predicted from the electrostatic simulation are shown as a curve on figure jub. Repeating the measurement for drift box potentials $200 \mathrm{~V}$ lower and higher, results in the residuals show $n$ in figure $b$. The data have been fit to a line with the same slope as before. The data agree nicely with the distortions predicted by the electrostatic simulation.

\section{Liquid radiator trays}

Operation of the liquid radiator trays requires good $\mathrm{CV}$ transmission of the liquid $\mathrm{C}_{6} \mathrm{~F}_{14}$ and quartz window. The quartz is checked upon receipt from the manufacturer in the same way as the drift box quartz.

We have built an apparat is to measure the UV transmission of liquid $C_{6} F_{14}$. This device is being used to conduct tests of how long the liquid retains transmission in the presence of the tray materials. We have begun by studying the transmission of the $\mathrm{C}_{6} \mathrm{~F}_{14}$ before exposure to the tray components. The liquid contains dissolved gases, particularly axygen, that must be removed to obtain good transmission. The $\mathrm{C}_{6} \mathrm{~F}_{14}$ test samples are degassed by several cycles of freezing in tiquid nitrogen, purging the container with boil-off $\mathrm{N}_{2} \mathrm{ges}$, and allowing the frozen liquid to thaw and equilibrate with the gas in the container. We have observed good transmission in the purified samples, although we are still studying the systematic limitations of our transmission measurements. When the tests are finished, the 'I' spectrometer will be moved to the SLD detector. There it will monitor the liquid at "arious stages as it is recirculated through the trays. including the output of any single tray.

It is essential that the liquid trays be strong enough to support the weight of the liquid and other smaller pressure 'ifferentials for the several-year life of the experiment. Two s. mple trays loaded with $\mathbf{C}_{6} F_{14}$ have been tested under slowly increasing pressure for many months [10]. The tests were performed at the CRID operating temperalure of $40^{\circ} \mathrm{C}$. The results are summarized in figure 7 . The first tray tested had a $3 \mathrm{~mm}$ thick window of ordinary foat glass. The second test tray had a $4 \mathrm{~mm}$ thick quartz window. the same thickness used in the production trays. For each tray, the window deflection was monitored throughout the test. The figure shous the observed window deflection for each tray as a function of the applied pressure, up to the breaking point. By comparing the deflections to those predicted by simple models of uniformly loaded plates with known boundary conditions [11], we have estimated the maximum stress in the window at the breaking point. In the case of the glass window, the deflections were well represented by a model in which the sides and rib of the tray serve as simple supports. The thicker quartz window is more closely reproduced by a model in which the rib is a fixed support. In both cases. the window failed at a stress of approximately $48 \mathrm{MPa}$ and in the region of maximum stress as predieted by the model. The quartz tray, having a thieler rindow, sustained a higher applied pressure. The quartz tray failed at a pressure of six times the largest load borne by any of the trays under normal operation.

\section{Mirrors}

The mirrors for the barre] CRID [12] are approximately 30 $\mathrm{cm} \times 30 \mathrm{~cm}$. with a radius of curvature 95.8 or $101.6 \mathrm{~cm}$. There are ten slightly different shapes of mirrors. Each of the ten mirrors directed on a drift box requires a shape specific to its position. The mirrors need to have an angular reflection error of less than 1 railliradian in order to matre a negligible contribution to the position error of the photoelectrons. They aiso need to have reflectivity of at least $85 \%$ in the wavelength range between 180 and 220 nm.

The optical distortion of each mirror has been measured photographically prior to applying the reflective coating by imaging a masl: placed in front of the mirror. The apparatus is such that a 1 milliradian reflection error corresponds to an error of approximately $1 \mathrm{~mm}$ on the negative. Only $3.5 \%$ of the mirror substrates failed this test and had to be replaced.

To obtain good reflectivity, the mirrors need both good surface smoothness and a good coaling. To keep light loss due to scattering to a few percent. the mirror substrates are polished $t o$ an average surface roughness of $\leq 3 \mathrm{~nm}$ 


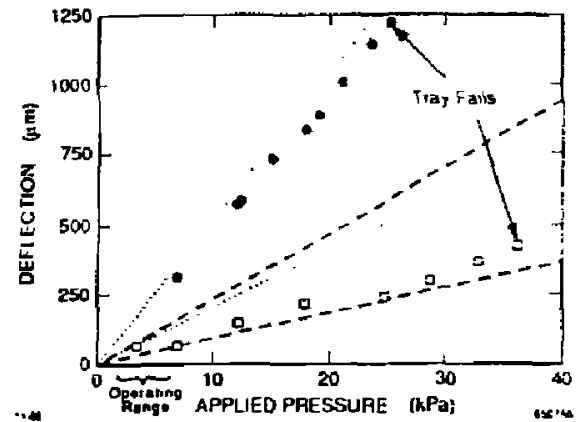

Figure 7 : Pressure tests of sample trays. The salid circles are for a tray with a 3 mm thick gless window. The two dotted lines show predictions for the model with simple support at the rib (steeper curve) and fixed support at the rib (lower rurve). The open squares and dashed lines show the data and predictions. respertively. for a tras with a 4 mm thick quartz window. The nornal operating range for the trays is $1.7-6.1 \mathrm{kPa}$.

(rms). The surface profile of approximately $70 \%$ of the mirrors was checked at Applied Magnetics Co.' with a surface profilometer. Seven percent of the mirrors failed the test and were replaced. Reflectivity of the coated mirrors relative to a standard sample was measured using a custom instrument (see [12]). Figure 8 shows the average reflectance of all the mirrors at eight wavelengths in the range $160-230 \mathrm{~nm}$. All of the mirrors satisfled the reflectivity requirements.

\section{Fluid Systems and Monitoring}

The CRID fluid delivery and monitoring systems are presently being assembled and tested. A comprehensive on-Jine system will continually monitor the purity, UV transpatency, electron lifecime, and mixing of the various fluids so that problems may be detected before the physits performance of the CRID is degraded. A more complite description of the monitor system can be found in ref. [13].

The $40^{\circ} \mathrm{C}$ operaling temperature of the CRJD is maintained using Kapton-insulazed heater pads attached to the surfaces of the vessel. The beaters are controlled according to the output of temperature sensors interspersed with the heaters, Current to the heaters is digitally switched using an $A C$ zero-crossing solid state relay. The relay is followed by a rectifier and a smoothing capacitor to provide "softstart" DC currene for use inside the SLD magnetic field. The total available heating power is $15 \mathrm{KW}$ at 150 volts

\footnotetext{
'Applied Magnetics Co., Goleta, CA 93117
}

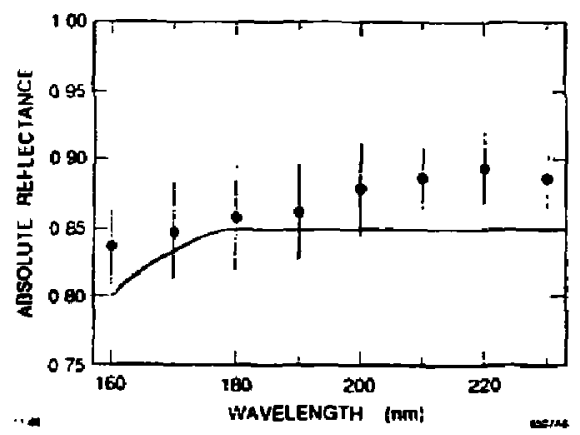

Higure 8. Average 1 : refectance measured for the production mirrors for the barrel CRID. Also shown is the minimum acceptable reflectance: $80 \%$ at $160 \mathrm{~nm}$, rising to $85 \%$ above $180 \mathrm{~nm}$.

DC. All of the gases and liquids circulating through the CRID will be preheated and delivered in heated lines.

Three gases are necessary for the operation of the CRID. The gas inside the drift box is ethane, bubbled through TMLAE at $28^{\circ} \mathrm{C}$ to achieve a concentration of about $0.1 \%$. It is then heated to $40^{\circ} \mathrm{C}$ for delivery to the drift boxes. The drift boxes are built with double side walls, separated by a purge space [14]. to help heep the electronegative radiator gas from entering the drift box or the gas laden with reaclive TMAE from escaping into the vessel volume. The purge volume is flushed with pure methane. Finally. the vessel is filled with gasorin: $C_{5} F_{12}$. The cast of the $C_{5} F_{12}$ requires that it be recirmiated. After flowing through the vessel. the gas is passed through filters to remove water, oxygen, and particulates before flowing through the vessel again. In addition. pore $\mathrm{N}_{\mathrm{y}}$ is used as a purge gas before or after opening the vessel to air. After sealing the vessel. it is first purged witn nitrogen until a satisfactory level of $\mathrm{O}_{2}$ and $\mathrm{H}_{2} \mathrm{O}$ is reached. The $\mathrm{N}_{2}$ is then thermodynamically replared with $C_{5} F_{12}$. Similarly, the $C_{5} F_{12}$ will be replaced by nitrogen before opening the vessel. The exchange is monitored by measuring the density of the gas with a sonar device [15]. both directly in the vessel and in the monitor system described below.

The drift gas, drift box purge gas, and radiator gas are all at atmospheric pressure. An analog system controls the pressure of each of the three systems by regulating the flow using a mass flow controller.? Adjustments are made based on the readings of capacitance manometers ${ }^{3}$ attached to each of the systems. Pressure differences between the drift box gas and radjator gas system must be small. no more than a few Torr, to prevent the pressure

\footnotetext{
2Modt] 258B. MKS lnc. Burlington, A1A 01803. L'SA.

${ }^{3}$ Model 221. MKS Inc., Burlington, MA 01803, USA.
} 
differences across the guartx drift box windows from cracking them. Similarly, deviations from atmospheric pressure must be small, so that pressure differences between the gas radiator and liquid radiator, which is separately referenced to at mosphere, do not jeopardize the liquid tray windows. The normal operating range for the gas systems is within 1 Tor of atmospheric pressure. The system has several levels of response to increasingly large pressure excursions, culminating in passive pressure relief through bubblers for deviations exceeding 5 Torr

We have made extensive provision for monitoring the quality of the gases delivered by the gas systems and also the gases returning from the CRID. This is shown schematically in figure 9. The upper part of the figure shows the system for checking source methane and ethane gas and the radiator gas. They can be checked for density (using the sonar), UV transmission, water content, and oxygen content. Samples can also be extracted for analysis in gas chromatograph. Similarly, gas containing TMAE from either of two TMAE bubblers and the output gas from the drift boxes and purge flow are examined by the system in the lower part of the drawing. Each drift box has a separate output line that can be selected. The output flow from the purge volumes is manifolded together to give one line for each half of the barrel. The gases connected to this part of the system can be checked in much the same way as the first group, except that the sonar is replaced by an electron lifetime monitor [7], which measures the electron attachment lifetime in the gas.

To reconstruct the production coordinate of an electron drifting over $1 \mathrm{~m}$ to an accuracy of $1 \mathrm{~mm}$, the drift velocity needs to be known to $0.1 \%$. The drift velocity will change during the day-to-day operation of the CRID, if for no other reason, then because of variations in atmospheric pressure. In addition to monitoring the pressure, the drift velocity will be measured directly in each drift box using fiber optics. Each box is equipped with 19 silica fibers 200 $\mu \mathrm{m}$ in diameter. They are all illuminated using a common $600 \mu \mathrm{m}$ fiber. The end of each fiber is held in a collimator glued to the quartz window on the outer (mirror) side of the box. UV light, from either a laser or a tash lamp, travels down the fibers and into the drift box, where it ionizes the TMAE. Comparing the arrival time of sigmals irom fibers al different known positions on the box will enable the drift velocity to be determined. The fibets will also be used to follow any time-dependent distortions in the drift field. One possible source of such a distortion would be positive jons accumulating on the sides, or windows, of the drift box.

Liquid $\mathrm{C}_{6} \mathrm{~F}_{14}$ for the radiator trays is provided by a recirculation system. The system maintains the $C_{6} F_{14}$ at $40^{\circ} \mathrm{C}$ throughout and uses only gravity to move the liquid through the trays. Spill-tanks (overflow devices) are used repeatedly at various levels to define and limit the liquid pressure heads at the trays and other critical points. The entire system is, at all levels, in contact with a buffer gas (purified $\mathrm{N}_{2}$ ), which serves as a pressure reference and, in turn, communicates with the atmosphete through a (cit: it condenser and bubblers. Backup blow-away bubblers are used to relieve the gas buffer system if there is a deviation from atmospheric pressure that could breal: the yuartz windows in the :rays. Pumps are used only to raise the liquid to the top of the system, whereupon it is dumped into a reservoir to begin its gravity descent.

Belore each cycle tbrough the trays. the liquid is pasiod through particulate filters, rater absorbents, and gasremoving ehemical columns. This guards against lass of LV transmission caused by buildup of $\mathrm{O}_{2}, \mathrm{H}_{2} \mathrm{O}$. or other impurities leaching from the trays or lines. The capacity is sized such that $120 \mathrm{cc} / \mathrm{s}$ of fluid can be delivered with $\$ 10 \mathrm{ppm}$ of contaminants of any given type, after initial purification of the $\mathrm{C}_{6} \mathrm{~F}_{14}, e . g$., by distillation and degassing. Montoring of all critical temperatures. pressures. flow rates, impurities and UV transmission are carried out continuously.

To monitor the fluid level in the trays to be sure that they fill completely, we have developed a simple technique using miniature light reflection emitter/sensor ${ }^{3}$ This device contains an infrared LED transmitter and a phototransistor. The conventional use of the sensor is to derect changes in the distance or reflectivity of surfaces in frons of it, with the highest sensitivity at a distance of ahout 1 $\mathrm{mm}$. The liquid $\mathrm{C}_{6} \mathrm{~F}_{14}$ is clear, and the bare sensor does not respond to being immersed in the liguid. However, we have obtained a nice signal to the presence of liquid if we opically couple the emitter and sensor with a dron of epoxy $y^{6}$ on the face of the device. Then, enough light is refiected from the surface of the drop to turn on the phototransistor. When the device is immersed in $C_{6} F_{1 .}$. less light is reflected at the interface between the epoxy and the liquid, reducing the current drawn by the transistor. The change in current, e.g., $1.4 \mathrm{~mA}$ dry to $04 \mathrm{~mA}$ wet, is easy to measure. Each tray is fitted with two of the epoxycoupled sensors, one each at the high and low corners of the tray, in the path of the fluid inlet and outlet. The low sensor will be used to confirm when flow has started to a tray, and tlie high sensor will check that the tray fills completely and remains full as liquid circulates.

\section{Conclusions}

The production of the components for the SLD barrel CRID is well along. with all of the mirrors completed and installed. We have described several tests which are designed to ensure the reliable operation of the components in the assembled CRID. Fluid and monitoring systems for the CRID are complicated by the $40^{\circ} \mathrm{C}$ operating temperature, the importance of all subsystems closely tracking atmospheric pressure, the high purity required, and the ne-

\footnotetext{
'Oxisarb Filters, Meseer Grieshein GMBH, D.4000 Düsseldurf 30. FRG.

${ }^{5}$ Catalog Mo. SFHgoo-2, Siemens Componenls, Inc.

'Equal parts by weight Epon 826 (Shell Co.) and Versamid 140 (Henkel Co.). Some other trangparent epoxies also work.
} 


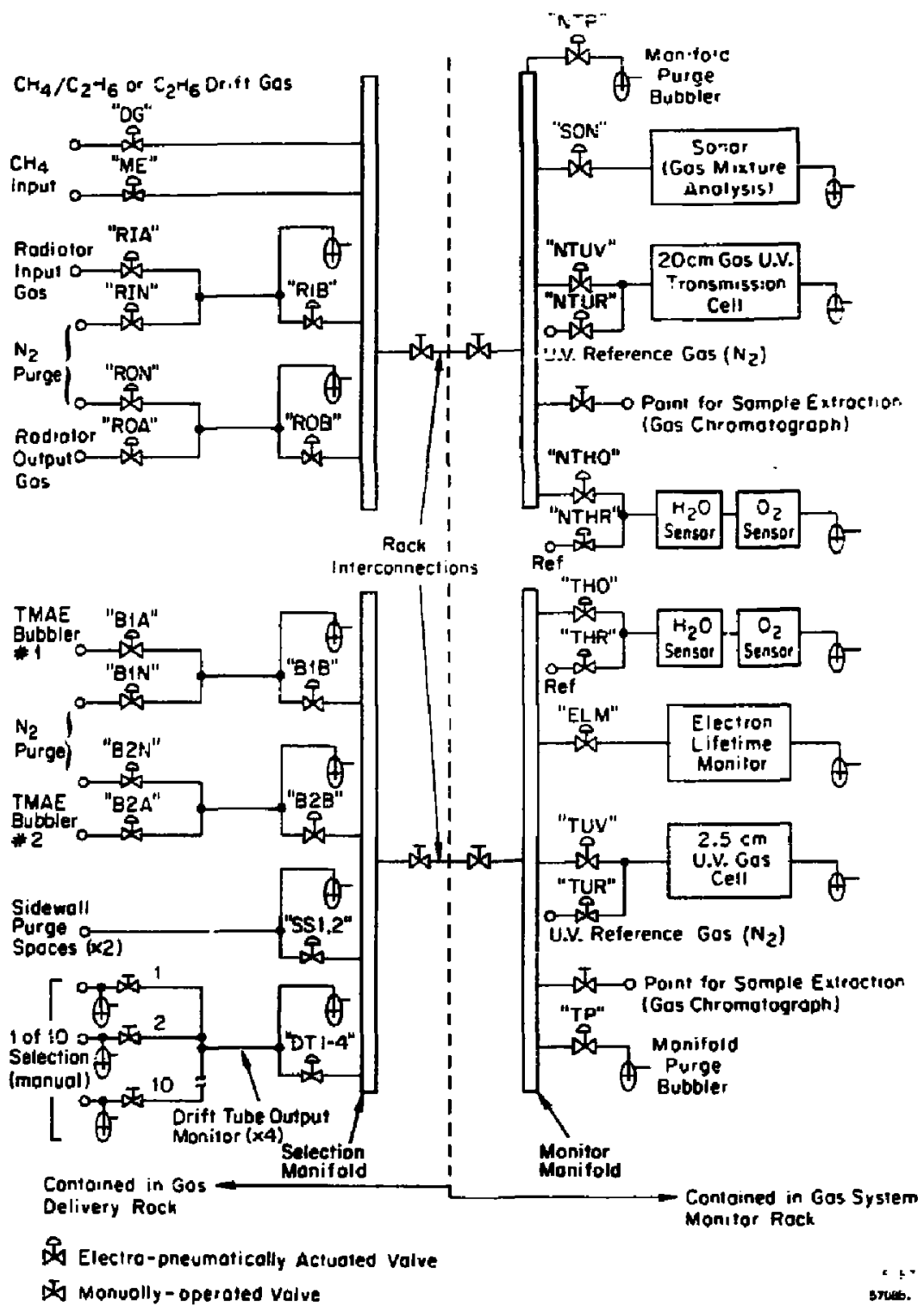

Figure 9: Schematic of the barrel C.RID gas monitor system. 
cessity of recycing the $C_{5} F_{12}$ gas and $C_{6} f_{14}$ liquid. These systcms are currently being assembled and tested.

\section{Acknowledgments}

The work described here would not have been possible without the assistance of the engineering and technica] stafts of the collaborating institutions. Their contributions are gratefully acknowledged. We thank $V$. Telnov for useful discusstons on the geometry of the drift field.

\section{References}

[1] SI.D Design Report SLAC-273 UC-34D, May 19s4, and revisions.

[2] D. Aston et al., SLAC-Pub-4795, Nucl. Instr. and sfeth., Sect. A, vol. 283, pp. 582-589, 1989.

[3] D. Aston ef al., SLAC-Pub-4875, Aivel. Instr, and Meth., Sect. A, vol. 283, pp. 590-595, 1989 ; see also 3. Va'vra et al., SLAC-Pub-4432, IEEE Trans. Nucl. Sci., vol. NS-35, Pp. 487-492, 1988, and ref. [4].

[4] D. Aston et al, SLAC-Pub-4785, IEEE T.ans. Nucl. Sci. vol. NS-36, pp. 276-282, 1989.

[5] E. Spencer at al., SLAC-Pub-4404, IEEE Trans. Nucl. Sci, vol. NS-35, pp. 231-236, 1988, and E. Spencer et al., "A Low Noise Preamplifier System for Cerenkov Ring Imaging Detectors," to be submitted to . Nuel. Instr. and Meth., Seci. A.

[6] R. T. Rewick et al., SLAC-Pub-4415, Anal. Chem., vol. 60 , p. 2095, 1988.

[7] M. Cavalli-Sforza. "Electron Lifetine Measurements in the Disaster Monitor," CRID Internal Note No. 15, 1986 .

[8] P. Coyle. et al., "Electron Drift Properties of the First Production Drift Boxes," CRID Internal Note No. 62. 1990.

[9] 5. Dasu, "Electrostatic Simulation of CRID Detector," CRID Internal Note No. 63, 1990.

[10] D. Coyne and D. Williams, "Strength and Breakage Tests of Liquid Radiator Trays," CRID Internal Note No. $61,1990$.

[11] See, e.g., S. Timoshenko and S. Woinowsky-Krieger, Theory of Plates and Shells, second ed., New York: McGraw-Hill, 1959.

[12] A. Bean et al., "Production of 400 Mirrors with High VUV Reflectivity for lse in a Ring Imaging Clierenkov Counter in SLI at SLAC." submitted to Nucl. Instr. and Meth., Sect. A.
[13] P. Antilogus et al., SLAC-Pub-5105. "Monitot and Control Systems for the SLD Cerenkov Ring Imag. ing Detector," contributed paper to the $\mathrm{II}^{-}$Confir. ence on Control Systems for Accelenators and Large Physics Expernments, Vancouver, Canada. Ortoher 29-November 3, 1989, to be published in Nuel Instr. and Meth., Sect. A.

[14] G. Hallewell el al., SLAC-Pub-4405, IEEE Trans. Siucl. Sci, vol. NS-35, pp. 398-403. 1988.

[15] G. Hallewell et al., SLAC-Pub-4122. Nucl. Instr. and Heth., Seet A, vol. 264, pp. 219-234, 1988

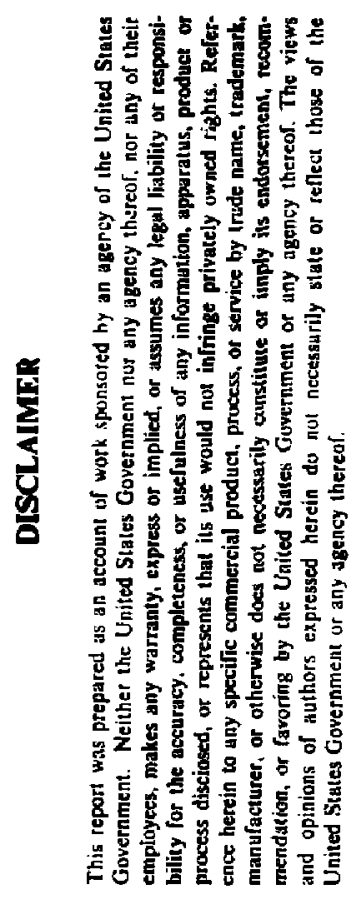

